\title{
Two spectroscopically confirmed galaxy structures at $z=0.61$ and 0.74 in the CFHTLS Deep 3 field ${ }^{\star}$
}

\author{
C. Adami ${ }^{1}$, E. S. Cypriano ${ }^{2}$, F. Durret ${ }^{3}$, V. Le Brun ${ }^{1}$, G. B. Lima Neto ${ }^{2}$, N. Martinet ${ }^{3}$, F. Perez ${ }^{1}$, \\ B. Rouze ${ }^{1}$, and L. Sodré Jr. ${ }^{2}$
}

\author{
1 Aix-Marseille Université, CNRS, LAM (Laboratoire d'Astrophysique de Marseille) UMR 7326, 13388 Marseille, France \\ e-mail: christophe.adami@lam.fr \\ 2 IAG, USP, R. do Matão 1226, 05508-090 São Paulo, SP, Brazil \\ 3 UPMC-CNRS, UMR 7095, Institut d’Astrophysique de Paris, 75014 Paris, France
}

Received 15 October 2014 / Accepted 16 December 2014

\section{ABSTRACT}

\begin{abstract}
Context. Galaxy evolution is known to depend on environment since it differs in clusters and in the field, but studies are sometimes limited to the relatively nearby Universe $(z<0.5)$. It is still necessary to increase our knowledge of cluster galaxy properties above $z=0.5$.

Aims. In a previous paper we have detected several cluster candidates at $z>0.5$ as part of a systematic search for clusters in the Canada France Hawaii Telescope Legacy Survey by applying the Adami \& MAzure Cluster FInder (AMACFI), based on photometric redshifts. We focus here on two of them, located in the Deep 3 (hereafter D3) field: D3-6 and D3-43.

Methods. We have obtained spectroscopy with Gemini/GMOS instrument and measured redshifts for 23 and 14 galaxies in the two structures. These redshifts were combined with those available in the literature. A dynamical and a weak lensing analysis were also performed, together with the study of X-ray Chandra archive data.

Results. Cluster D3-6 is found to be a single structure of eight spectroscopically confirmed members at an average redshift $z=0.607$, with a velocity dispersion of $423 \mathrm{~km} \mathrm{~s}^{-1}$. It appears to be a relatively low-mass cluster. D3-43-S3 has 46 spectroscopically confirmed members at an average redshift $z=0.739$. The cluster can be decomposed into two main substructures, having a velocity dispersion of about 600 and $350 \mathrm{~km} \mathrm{~s}^{-1}$. An explanation of the fact that D3-43-S3 is detected through weak lensing (only marginally, at the $\sim 3 \sigma$ level) but not in X-rays could be that the two substructures are just beginning to merge more or less along the line of sight. We also show that D3-6 and D3-43-S3 have similar global galaxy luminosity functions, stellar mass functions, and star formation rate (SFR) distributions. The only differences are that D3-6 exhibits a lack of faint early-type galaxies, a deficit of extremely high stellar mass galaxies compared to D3-43-S3, and an excess of very high SFR galaxies.

Conclusions. This study shows the power of techniques based on photometric redshifts to detect low to moderately massive structures, even at $z \sim 0.75$. Combined-approach cluster surveys such as EUCLID are crucial to find and study these clusters at these relatively high redshifts. Finally, we show that photometric redshift techniques are also well suited to study the galaxy content and properties of the clusters (galaxy types, SFRs, etc.).
\end{abstract}

Key words. galaxies: clusters: general

\section{Introduction}

Galaxy evolution is still a major topic in cosmology because of the complexity of the processes involved. The current theoretical framework (e.g. White \& Frenk 1991) assumes that galaxies are formed by accretion of baryonic matter onto dark matter haloes and evolve through mergers and other interactions with other galaxies and the intergalactic medium (IGM). The study of evolution in clusters is particularly interesting because some extreme conditions (e.g. high galaxy density, large amounts of hot gas in the IGM) are found in these structures and, consequently, galaxy evolution is affected by many environmentallydriven processes, as evidenced by the evolution of the morphological mix of their galaxies (e.g. Boselli et al. 2014). Naturally, in this scenario the evolution of galaxies is not independent from the evolution of cluster structures. According e.g. to Mateus et al. (2007), galaxy evolution is accelerated in denser environments. All this makes clusters ideal laboratories to investigate galaxy evolution.

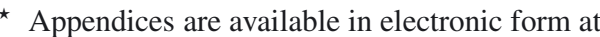
http: //www . aanda.org
}

Most of our current knowledge about processes affecting galaxies in clusters comes primarily from studies in the nearby $(z<0.5)$ Universe. At higher redshifts, several studies have focussed on the evolution of morphological type fractions (e.g. Holden et al. 2007; van der Wel et al. 2007; Simard et al. 2009), on their clustering properties (e.g. Poggianti et al. 2010; Ross et al. 2010), or on the evolution of specific galaxy types (such as red galaxies: Sánchez-Blázquez et al. 2009).

The question we want to answer is how do cluster galaxies evolve in the redshift interval $0.5<z<1$. We propose adding our contribution to this problem through spectroscopy and photometry of candidate cluster galaxies observed with GEMINI/GMOS and primarily detected from CFHTLS survey photometric redshift catalogues. In this framework, Adami et al. (2010) have published a catalogue of $\sim 1200$ cluster candidates from public photometric redshifts (obtained following Ilbert et al. 2006; also see Coupon et al. 2009) of the CFHTLS data release T0004 in the D2, D3, D4, W1, W3, and W4 regions. This catalogue contains several cluster candidates at redshift larger than 0.5 , detected with high confidence (signal-to-noise ratio equal to or larger than 4) and also detected by other methods 
(Olsen et al. 2007; Thanjavur et al. 2009). We choose to focus on two of them.

We describe the optical identification of our candidate clusters in Sect. 2. The mass characterisation of the two structures is discussed in Sect. 3. In Sect. 4 we present some properties of the galaxy populations in the clusters, and in Sect. 5 we summarise our results.

We adopted, where necessary, the Dunkley et al. (2009) $\Lambda \mathrm{CDM}$ concordance cosmological model $\left(H_{0}=\right.$ $71.9 \mathrm{~km} \mathrm{~s}^{-1} \mathrm{Mpc}^{-1}, \Omega_{\Lambda}=0.742, \Omega_{\mathrm{M}}=0.258$ ).

\section{Optical identification of our candidate clusters}

\subsection{CFHTLS imaging}

The two clusters studied here were discovered as part of a systematic search for clusters in the Canada France Hawaii Telescope Legacy Survey (Adami et al. 2010). They were found in the Deep 3 field, which covers $1 \mathrm{deg}^{2}$ and is centred at coordinates 14:19:27.00, +52:40:56 (J2000.0). This field is among the deepest optical images available from the ground, corresponding to a total exposure time of the order of $90 \mathrm{~h}$.

To identify clusters in this field we applied the Adami \& MAzure cluster FInder (AMACFI, Mazure et al. 2007), based on photometric redshifts (hereafter photo-zs). After the photo$z$ catalogue is cut in photometric redshift slices, galaxy density maps are drawn with an adaptive kernel technique, and overdensities are detected at a chosen significance level with SExtractor. Detections are then sorted with a minimal spanning tree method, leading to a catalogue of candidate clusters.

The two candidate clusters studied here were detected at a $4 \sigma$ significance level. Their identifications in the Adami et al. (2010) catalogue are D3-6 and D3-43.

\subsection{Gemini GMOS spectroscopy}

We were awarded $8.5 \mathrm{~h}$ of GEMINI/GMOS time (program GN-2011A-Q46, PI: L. Sodré) to observe these two cluster candidates spectroscopically. We initially used the R400 grism and one arcsec slits. Final 1D spectra were rebinned to have $\sim 7 \AA$ per pixel.

We were able to fit 27 and 16 slits in the D3-6 and D3-43 fields respectively. Reduction was made in the IRAF environment with the GMOS dedicated tools. We applied the EZ redshift measurement code (Garilli et al. 2010) on the final 1D spectra, allowing an additional smoothing from 3 to 9 pixels to find the redshift value more easily. The redshift measurements were done in the same way as for the VIPERS survey (e.g. Guzzo et al. 2014). Independent measurers provided two first estimates of the redshifts (Le Brun \& Adami). The two values were then reconciled and a quality flag was assigned between 1 and 4 . Flag 1 means that we have a $50 \%$ chance to have the correct redshift estimate, flag 2 means that we have a $75 \%$ chance, flag 3 means that we have a $95 \%$ chance, and flag 4 means that we have more than $99 \%$ chance. We only considered the objects with flags 2,3 , and 4 to be successful measurements. We obtained an excellent success rate: 15 of the 16 D3-43 spectra and 26 of the 27 D3-6 spectra provided successful redshift measurements. Only four spectra turned out to be of stellar origin, while we obtained galaxy redshifts for 23 and 14 galaxies in the fields of D3-6 and D3-43, respectively.

Coordinates and successfully measured redshifts for this sample are given in Tables 1 and 2 . We show four examples of spectra corresponding to flags 4, 3, and 2 in Figs. B.1 and B.2.
Table 1. Running number, coordinates (J2000), redshifts, and spectral flags for the objects observed with GEMINI/GMOS in the D3-43 field of view.

\begin{tabular}{rrrrr}
\hline \hline$\#$ & RA & Dec & Redshift & Flag \\
\hline 1 & $14: 20: 51.079$ & $53: 03: 38.76$ & 0.8264 & 3 \\
2 & $14: 20: 56.214$ & $53: 02: 21.28$ & 0.8950 & 3 \\
3 & $14: 20: 56.472$ & $53: 02: 35.13$ & 0.7111 & 4 \\
4 & $14: 20: 58.824$ & $53: 01: 11.63$ & 0.7497 & 4 \\
5 & $14: 20: 59.161$ & $53: 01: 36.41$ & 0.7404 & 4 \\
6 & $14: 21: 00.276$ & $53: 04: 29.72$ & 0.7737 & 4 \\
7 & $14: 21: 02.344$ & $53: 05: 03.26$ & 0.6723 & 4 \\
8 & $14: 21: 02.572$ & $53: 01: 22.74$ & 0.3347 & 4 \\
9 & $14: 21: 05.073$ & $53: 02: 06.92$ & 0.7393 & 4 \\
10 & $14: 21: 10.762$ & $53: 02: 49.93$ & 0.7350 & 4 \\
11 & $14: 21: 11.665$ & $53: 04: 44.20$ & 0.8217 & 3 \\
12 & $14: 21: 15.013$ & $53: 00: 57.71$ & 0. & 4 \\
13 & $14: 21: 18.394$ & $53: 03: 28.41$ & 0.9748 & 4 \\
14 & $14: 21: 18.842$ & $53: 00: 32.05$ & 0.7395 & 4 \\
15 & $14: 21: 21.818$ & $53: 01: 55.12$ & 0.7321 & 4 \\
\hline
\end{tabular}

Table 2. Running number, coordinates (J2000), redshifts, and spectral flags for the objects observed with GEMINI/GMOS in the D3-6 field of view.

\begin{tabular}{rrrrr}
\hline \hline$\#$ & RA & Dec & Redshift & Flag \\
\hline 1 & $14: 16: 36.004$ & $53: 04: 31.73$ & 0.7163 & 3 \\
2 & $14: 16: 37.152$ & $53: 04: 32.32$ & 0.5268 & 3 \\
3 & $14: 16: 38.064$ & $53: 03: 44.66$ & 0.7441 & 2 \\
4 & $14: 16: 39.084$ & $53: 05: 44.23$ & 0.6083 & 4 \\
5 & $14: 16: 40.173$ & $53: 05: 01.37$ & 0. & 4 \\
6 & $14: 16: 41.339$ & $53: 04: 54.53$ & 0.6474 & 3 \\
7 & $14: 16: 46.077$ & $53: 01: 47.67$ & 0.5758 & 4 \\
8 & $14: 16: 46.824$ & $53: 04: 38.55$ & 0.6090 & 4 \\
9 & $14: 16: 47.833$ & $53: 03: 04.95$ & 0.6046 & 4 \\
10 & $14: 16: 49.100$ & $53: 05: 55.98$ & 0.6067 & 3 \\
11 & $14: 16: 50.122$ & $53: 05: 10.06$ & 0.6077 & 3 \\
12 & $14: 16: 51.068$ & $53: 02: 17.15$ & 0.6007 & 2 \\
13 & $14: 16: 53.373$ & $53: 05: 51.77$ & 0. & 4 \\
14 & $14: 16: 55.542$ & $53: 05: 16.50$ & 0.6064 & 4 \\
15 & $14: 16: 56.983$ & $53: 02: 43.27$ & 0.9006 & 3 \\
16 & $14: 16: 57.645$ & $53: 04: 29.12$ & 0.6074 & 2 \\
17 & $14: 16: 58.438$ & $53: 03: 47.13$ & 0.5888 & 2 \\
18 & $14: 16: 59.666$ & $53: 02: 26.51$ & 0.6064 & 4 \\
19 & $14: 17: 00.703$ & $53: 05: 15.49$ & 0.9670 & 4 \\
20 & $14: 17: 01.736$ & $53: 03: 19.29$ & 0.6441 & 3 \\
21 & $14: 17: 03.493$ & $53: 00: 50.72$ & 0.5255 & 2 \\
22 & $14: 17: 04.775$ & $53: 03: 15.64$ & 0.7748 & 4 \\
23 & $14: 17: 05.915$ & $53: 05: 58.64$ & 0.5255 & 3 \\
24 & $14: 17: 06.757$ & $53: 05: 55.87$ & 0.5270 & 4 \\
25 & $14: 17: 08.225$ & $53: 00: 47.94$ & 0.6478 & 4 \\
26 & $14: 17: 09.578$ & $53: 03: 34.14$ & 0. & 4 \\
\hline
\end{tabular}

\subsection{Publicly available spectroscopy}

One of the two studied candidate clusters (D3-43) was also covered by the KECK/DEIMOS DEEP2 spectroscopic survey (Newman et al. 2013). This provided 336 redshifts in a 5 arcmin radius circle around the cluster centre, in addition to the GEMINI/GMOS redshifts. Ten of these GEMINI/GMOS redshifts were also measured by the KECK/DEIMOS DEEP2 survey, and this allowed us to assess our own redshift measurements, as shown in Fig. A.1. We do not detect any noticeable difference. Even the two galaxies with the most different redshift values in the two surveys do not exhibit a redshift difference larger than 0.015 . This typically corresponds to the $3 \sigma$ uncertainty according to the smoothing we applied to 
Table 3. Structures detected along the line of sight to the two candidate clusters.

\begin{tabular}{lrrrrr}
\hline \hline Name & $\#$ & $N_{\text {gal }}$ & $z$ & Vel. disp. $\left(\mathrm{km} \mathrm{s}^{-1}\right)$ & SG substructures \\
\hline D3-6 & 1 & 8 & 0.607 & 423 & 1 \\
\hline D3-43 & S1 & 17 & 0.201 & 489 & $/$ \\
& S2 & 11 & 0.378 & 250 & $/$ \\
& S3 & 46 & 0.739 & 1152 & 2 \\
& S4 & 18 & 0.975 & 575 & $/$ \\
\hline
\end{tabular}

Notes. The columns are: (1) cluster name; (2) number of the structure; (3) number of galaxies in the structure; (4) mean redshift of the structure; (5) Rostat velocity dispersion; (6) number of substructures when analysed by the Serna \& Gerbal (1996) method.

GEMINI/GMOS spectra before redshift measurements (dashed lines in Fig. A.1). If we consider only redshifts lower than 0.8, the typical uncertainty between the two redshift measurements is 0.0005 .

The other candidate cluster (D3-6) has only three additional objects with known spectroscopic redshifts available through the NED and Simbad databases (Howell et al. 2005; Hsieh et al. 2005; Walker et al. 2011). None are in common with our GEMINI/GMOS catalogue.

\subsection{The main structures along the two lines of sight}

We first merged our own redshift catalogue with those of the literature and eliminated the galaxies observed twice, as well as the stars. We then computed redshift histograms along the two lines of sight, and searched for empty gaps wider than 0.001 in the redshift distribution. This is a classical method to detect galaxy structure candidates (e.g. Katgert et al. 1996). We only retained galaxy concentrations between two such gaps of more than five objects. This provided four potential structures along the D3-43 line of sight and only one along the D3-6 line of sight (see Table 3 and Fig. 1).

The D3-6 line of sight only shows one structure at $z=0.607$ (see Fig. 2) sampled with eight galaxies with spectroscopic redshifts. The $\left(r^{\prime}-z^{\prime}\right)$ values of these galaxies are relatively similar and of the order of 1.4 and 1, also in good agreement with the colours of $z=0.5$ early-type and spiral galaxies respectively (e.g. Fukugita et al. 1995). The mean redshift of the only detected structure along the D3-6 line of sight $(z=0.607)$ also being close to the candidate cluster photometric redshift value $(z=0.6)$, we decided to associate it with the D3-6 candidate cluster of Adami et al. (2010).

Figure 3 displays the colour-magnitude relations of the potential structures along the D3-43 line of sight in the $\left(r^{\prime}-z^{\prime}\right)$ versus $r^{\prime}$ space. We clearly see a red sequence (showing an old galaxy population) around $\left(r^{\prime}-z^{\prime}\right) \sim 1.8$ and a bluer cloud around $\left(r^{\prime}-z^{\prime}\right) \sim 1$ for structure S3. These colours are consistent with $z=0.8$ early and late-type galaxies (e.g. Fukugita et al. 1995). Other structures of the D3-43 line of sight are less prominent and do not show the same dichotomy between an early-type and a late-type galaxy population. The structure S3 is also by far the most populated: it has more confirmed galaxies than the three others taken together. The structure S3 is clearly the closest to the $3 \sigma$ peak of the weak lensing detection (see below and Fig. 6). The brightest galaxy of S3 is at $\sim 600 \mathrm{kpc}$ of the peak, while the brightest galaxies of S1, S2, and S4 are located at $\sim 850 \mathrm{kpc}$, $\sim 2300 \mathrm{kpc}$, and $\sim 1300 \mathrm{kpc}$ (at the structure redshift). The structure $\mathrm{S} 3$ is the only structure for which the galaxy dispersion on the sky is larger than the distance between the structure centre
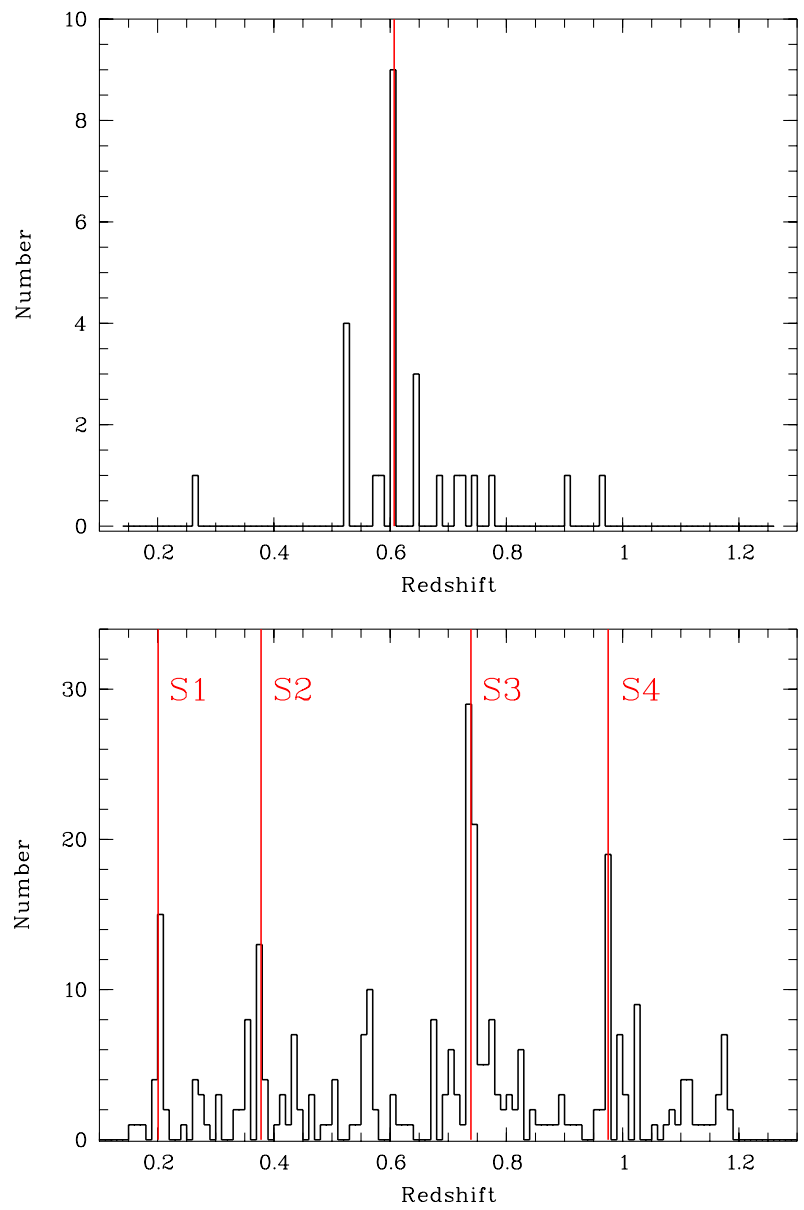

Fig. 1. Distribution of the Gemini/GMOS and DEEP2 galaxies with known spectroscopic redshifts along the D3-6 (top), and D3-43 (bottom) lines of sight. Detected structures from Table 3 are shown as red vertical lines.

and the $3 \sigma$ peak of the weak lensing detection $(1.2 \times$ larger $)$. The ratio is 0.4 for $\mathrm{S} 1,0.3$ for $\mathrm{S} 2$, and 0.9 for $\mathrm{S} 4$. Finally, the spectroscopic redshift of S3 is remarkably close to the photometric redshift of D3-43 in Adami et al. (2010): 0.75. We therefore decided to choose S3 as the main structure along the D3-43 line of sight, to associate it with the weak lensing detection (see below), and with the D3-43 candidate cluster of Adami et al. (2010).

This leads us to conclude that we probably have detected at least two real structures along the D3-6 and D3-43 lines of sight at $z=0.607$ (D3-6 in the following) and $z=0.739$ (D3-43-S3 in the following) respectively. The redshift distributions of the galaxies with known spectroscopic redshifts inside these structures are displayed in Fig. 4.

\subsection{Internal structure of D3-6 and D3-43-S3}

We investigated the internal structures of D3-6 and D3-43-S3 by applying the Serna-Gerbal technique (hereafter SG, 1996 release, Serna \& Gerbal 1996). This hierarchical code based on spectroscopic redshifts and optical magnitudes, is designed to detect substructures in the optical. A number of other methods are available to search for substructures at optical wavelengths, such as the $\Delta$-test (Dressler \& Schechtman 1988). However, the SG method has proven to be quite powerful detecting evidence for substructuring in nearby (see Abell 496: Durret et al. 2000; Coma: Adami et al. 2005, 2009; Abell 780: Durret et al. 2009; Abell 85: Boué et al. 2008), moderate redshift 
Table 4. Main characteristics of the two confirmed structures.

\begin{tabular}{lrrrrrrrrr}
\hline \hline Name & $\begin{array}{r}\text { RA(D3) } \\
\text { deg }\end{array}$ & $\begin{array}{r}\text { Dec(D3) } \\
\text { deg }\end{array}$ & $z$ (D3) & $\begin{array}{r}\text { RA(spec) } \\
\text { deg }\end{array}$ & $\begin{array}{r}\text { Dec(spec) } \\
\text { deg }\end{array}$ & $\begin{array}{r}z \text { (spec }) \\
\text { Vel. disp. } \\
\mathrm{km} \mathrm{s}^{-1}\end{array}$ & $\begin{array}{r}\text { SG mass } \\
M_{\odot}\end{array}$ & $\begin{array}{c}\text { X-ray mass } \\
M_{\odot}\end{array}$ \\
\hline D3-6 & 214.2207 & 53.0568 & 0.60 & 214.2314 & 53.0879 & 0.607 & 423 & $2.2 \times 10^{14}$ & $2.5 \times 10^{14}$ \\
D3-43-S3 & 215.271 & 53.0353 & 0.75 & 215.2710 & 53.0353 & 0.739 & $1152(611+357)$ & $3.6 \times 10^{14}+8.8 \times 10^{13}$ & $\leq 8 \times 10^{13}$ \\
\hline
\end{tabular}

Notes. The columns are: (1) name; (2) + (3) coordinates in the D3 CFHTLS cluster catalogue; (4) photometric redshift in the D3 CFHTLS cluster catalogue; (5) and (6) central coordinates of the spectroscopic catalogue defined as the coordinates of the brightest galaxy member; (7) mean spectroscopic redshift; (8) velocity dispersion from Rostat estimates (Beers et al. 1991; values are also given for the two substructures of D3-43-S3); (9) mass estimate from the Serna-Gerbal analysis (values are also given for the two substructures of D3-43-S3); (10) mass estimate from the X-ray analysis (within $r_{500}$ ).

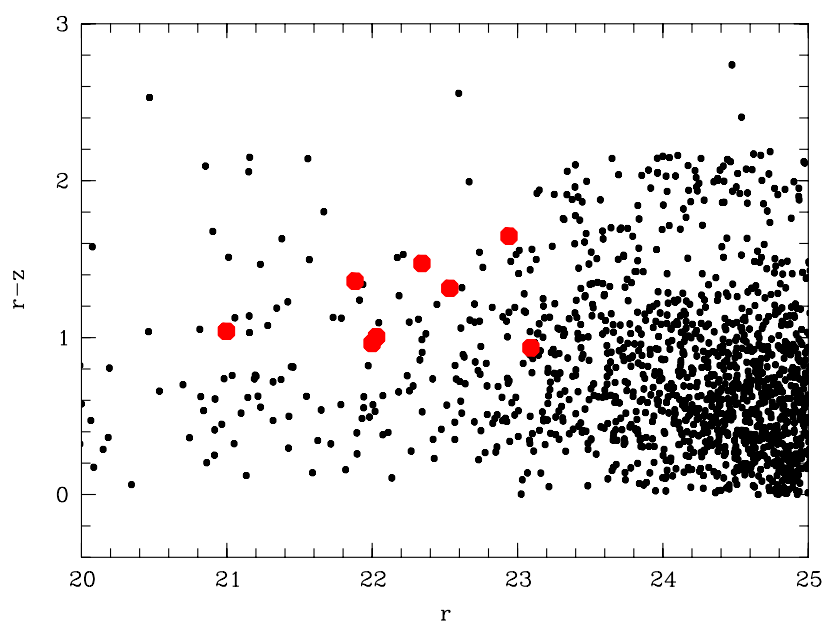

Fig. 2. Colour-magnitude relation along the D3-6 cluster line of sight. Small black dots are the CFHTLS galaxies, red disks are the spectroscopically confirmed cluster members.

(Abell 222/223: Durret et al. 2010), and high redshift clusters (RX J1257.2+4738: Ulmer et al. 2009). Recently, it was also successfully applied and compared with X-ray detections on a larger sample (the DAFT/FADAS sample: Guennou et al. 2014). Assuming a value of the mass to luminosity ratio (here taken to be 100 in the $r^{\prime}$ band, to be homogeneous with the Guennou et al. 2014 simulations), the SG method allows us to estimate the masses of the substructures that it detects. Guennou et al. (2014) have shown that although the absolute masses are not accurate (typical uncertainties are clearly larger than $10^{14} M_{\odot}$ ), the mass ratios of the various substructures were well determined. The SG method has also been extensively tested on simulations by Guennou (2012), in particular concerning the effect of undersampling on mass determinations. We stress that this method requires a very good precision in the galaxy distance determinations, and therefore spectroscopic redshifts are essential, while photometric redshifts are inappropriate.

The level of refinement in the substructure detection obviously depends on the spectroscopic sampling, as already shown by the previously quoted articles. We first processed the D3-6 structure. Since it is only sampled by eight galaxies with known spectroscopic redshifts, the SG code would only be able to detect large substructures that would be present in clustercluster merging, and this is not the case, showing that D3-6 is probably not undergoing a major merger.

The D3-43-S3 structure is much better sampled spectroscopically. The SG method detects two relatively massive substructures (see Table 4) and several other more dynamically isolated galaxies. Substructure \#1 includes the dominant galaxy of
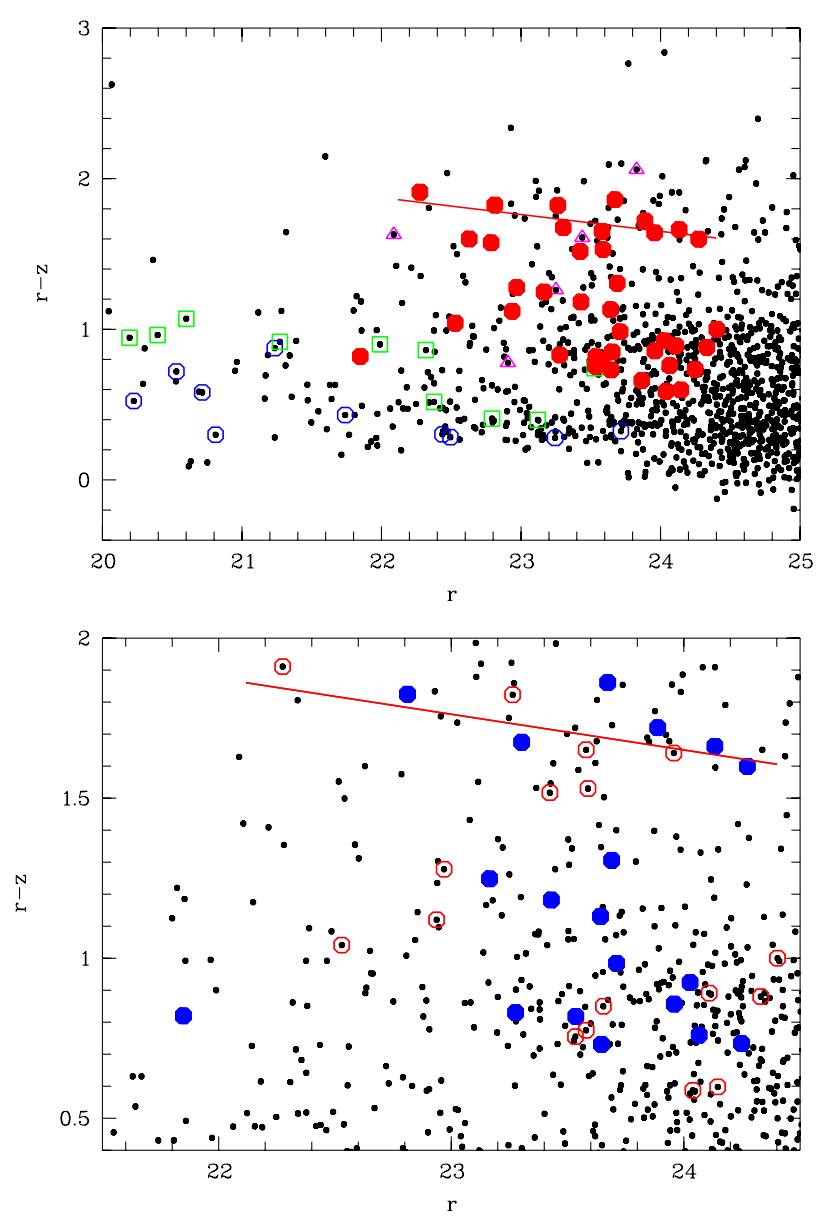

Fig. 3. Colour-magnitude relations along the D3-43 candidate cluster line of sight. Small black dots are the CFHTLS galaxies. Upper figure: red disks are the spectroscopically confirmed members of $\mathrm{S} 3$, blue circles are the S1 members, green squares are the S2 members, pink triangles are the $\mathrm{S} 4$ members. The inclined red line is the tentative red sequence for structure S3 early-type galaxies. Lower figure: red circles are the $\mathrm{S} 3$ substructure \#1 members, blue disks are the $\mathrm{S} 3$ substructure \#2 members (see text for details). The inclined red line is the tentative red sequence for $\mathrm{S} 3$ early-type galaxies.

D3-43-S3 and has an estimated dynamical mass of 3.6 $\times 10^{14} M_{\odot}$. The estimated mass of substructure \#2 is lower, of the order of $8.8 \times 10^{13} M_{\odot}$. D3-43-S3 is therefore probably about to undergo an important merger between at least two comparable galaxy structures (see also Sect. 5). To confirm this statement, we also show in Fig. 3 (bottom part) the colour-magnitude relation of the two substructures in the D3-43-S3 region. This shows that the two substructures have both red and blue galaxies. We are 

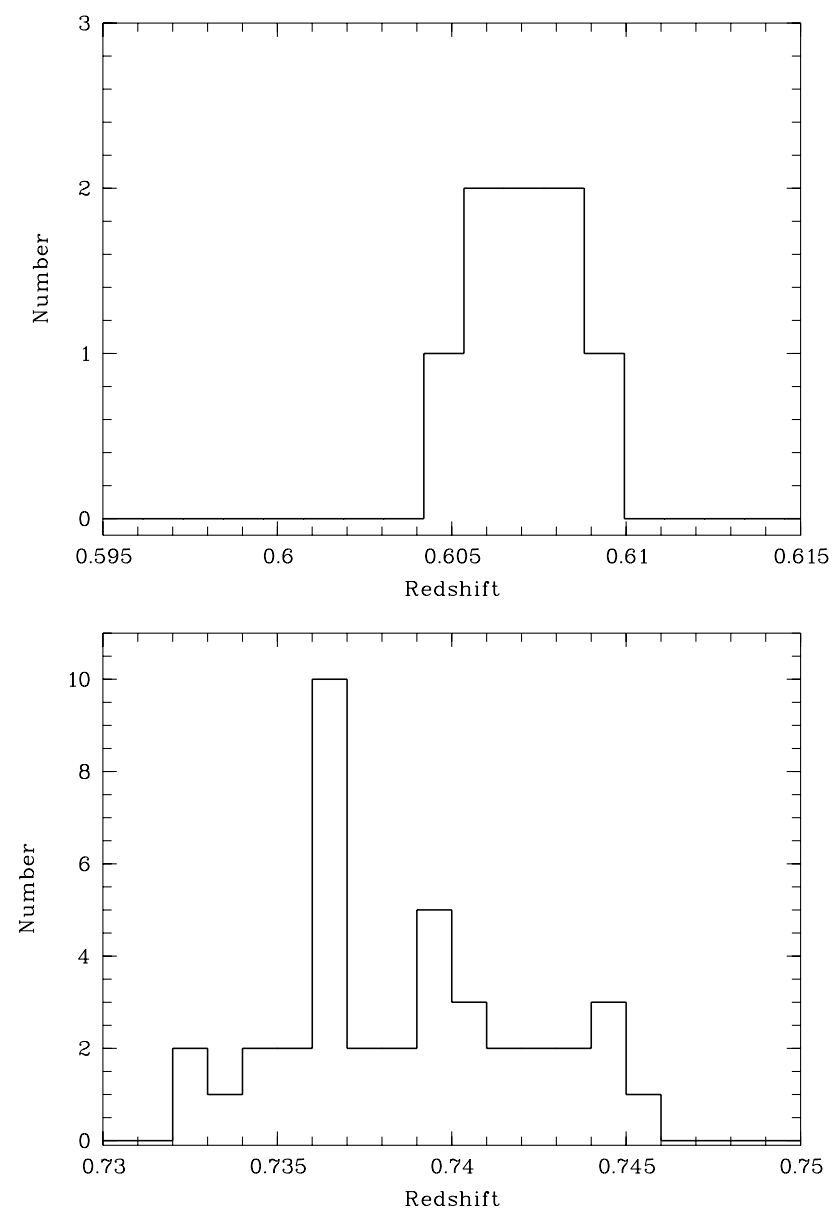

Fig. 4. Redshift distribution of the Gemini/GMOS galaxies with known spectroscopic redshifts in the D3-6 (top), and D3-43-S3 (bottom) structures.

therefore not dealing with a structure populated by young galaxies merging with an older galaxy structure. The apparently high velocity dispersion of D3-43-S3 (when not considering the detected substructures) is also consistent with this scenario.

\section{Mass characterisation of the D3-6 and D3-43-S3 structures}

At this stage, we have secured the detection of the D3-6 and D3-43-S3 structures, confirmed that they are populated by both red and blue galaxies, shown that D3-6 is not undergoing a major merger, and that D3-43-S3 is probably about to undergo such a merger. However, we do not yet have robust estimates of the masses of these two structures (the SG test does not give reliable absolute mass values, as already stated).

\subsection{Publicly available X-ray data}

Both fields D3-6 and D3-43-S3 were observed by the Chandra $\mathrm{X}$-ray telescope. Even if the collecting area of Chandra is not favourable to the characterisation of such distant structures, the exposure times were long enough to at least give an estimate of the X-ray luminosities of these structures.

There are four pointings at the D3-43 region with the ACIS-I detector (obs_id 5845, 5846, 56214, 6215, PI K. Nandra, taken in 2005). Following the "Science Threads" from the Chandra
X-ray Center (CXC), using CIAO $4.6^{1}$, we have reprocessed these observations and merged them together producing a single broad-band (0.5-7.0 keV) exposure-map corrected surface brightness image with a pixel scale of $1.968 \operatorname{arcsec}\left(14 / h_{70} \mathrm{kpc}\right.$ assuming $z=0.739$ ). The total effective exposure time for this image is $193.6 \mathrm{ks}$. There is apparently no visible large scale extended X-ray emission coinciding with the position of D3-43-S3 (e.g. not detected in Erfanianfar et al. 2013). We only see a collection of three compact sources. In a circular region of radius $R=2$ arcmin, we estimate that an extended source with count rate above $4.2 \times 10^{-4} \mathrm{cnt} / \mathrm{s}$ (about 85 counts) would be detected. Thus, assuming a plasma thermal emission, we can put an upper limit of $f_{\mathrm{X}}<4.3 \times 10^{-15} \mathrm{erg} \mathrm{s}^{-1} \mathrm{~cm}^{-2}$. This corresponds to an upper limit for the X-ray luminosity $L_{\mathrm{X}}<1.5 \times 10^{43} \mathrm{erg} \mathrm{s}^{-1}$ if the source is at $z=0.739$. This could be typical of a massive structure of galaxies, but at this stage this is not enough to reach a conclusion about the massive nature of D3-43-S3.

The D3-6 region was observed in April 2002 with ACIS-I (obs_id 3239, P.I. E. Ellingson) with an exposure time of $62.82 \mathrm{ks}$. We followed the same reduction procedure, producing a flat (exposure-corrected) image in the $0.5-7.0 \mathrm{keV}$ band with a pixel scale of 1.968 arcsec $\left(13 / h_{70} \mathrm{kpc}\right.$ assuming $z=0.607)$. A faint, extended source is visible at 14:17:01.7, $+53: 05: 13$ (J2000). To produce an image showing only the diffuse component, we have detected all point sources with the task WAVDETECT, following CIAO Science Threads ${ }^{2}$, which is based on the wavelet image decomposition technique. Then, the regions containing the detected point sources were replaced with a Poissonian noise, using the DMFILTH task, with the same mean value sampled from an elliptical annulus around the source. Finally, for display purposes, we have smoothed the diffuse emission image with a Gaussian kernel of 12 pixels (23.6"). Within 2 arcmin, we estimate a net count rate (background subtracted) of $(5.56 \pm 0.97) \times 10^{-3} \mathrm{cnt} / \mathrm{s}$. This corresponds to a flux $f_{\mathrm{X}}=(5.44 \pm 0.92) \times 10^{-14} \mathrm{erg} \mathrm{s}^{-1} \mathrm{~cm}^{-2}$ and, assuming a redshift $z=0.607$, a bolometric luminosity $L_{\mathrm{X}}=(1.12 \pm 0.19) \times 10^{44} \mathrm{erg} \mathrm{s}^{-1}$, typical of a low-mass cluster of galaxies. The derived X-ray luminosity corresponds to a velocity dispersion $\sigma_{v} \approx 500 \mathrm{~km} \mathrm{~s}^{-1}$, using the scaling relation by Lopes et al. (2009). This estimate is very close to our SernaGerbal value (see Table 4). We show in Fig. 5 the Chandra contours and the galaxies with known spectroscopic redshifts in the D3-6 cluster overlaid onto the CFHTLS $i^{\prime}$ band image. Five of the galaxies with known spectroscopic redshifts are at less than $1 \mathrm{Mpc}$ from the cluster X-ray centre, and this is probably enough to secure the association between the X-ray emission and the galaxy redshift concentration (see e.g. Adami et al. 2011, for the XMM-LSS survey). This association would need to be definitively confirmed by measuring the redshift of the X-ray central galaxy, however.

If we convert the X-ray luminosities to masses by applying the scaling relation of Lopes et al. (2009), we find a mass of $2.5 \times 10^{14} M_{\odot}$ for D3-6, and an upper limit of $8 \times 10^{13} M_{\odot}$ for D3-43 (calculated within $r_{500}$ ).

\subsection{Weak lensing characterisation of D3-43-S3}

To check if we can investigate the mass distribution around D3-43-S3 in an independent way, we took advantage of the CFHTLS D3 images in the $i^{\prime}$ band for which a special release is available with a seeing of 0.64 arcsec. Even with such a good

\footnotetext{
1 asc.harvard.edu/ciao/

2 http://cxc.harvard.edu/ciao/threads/
} 


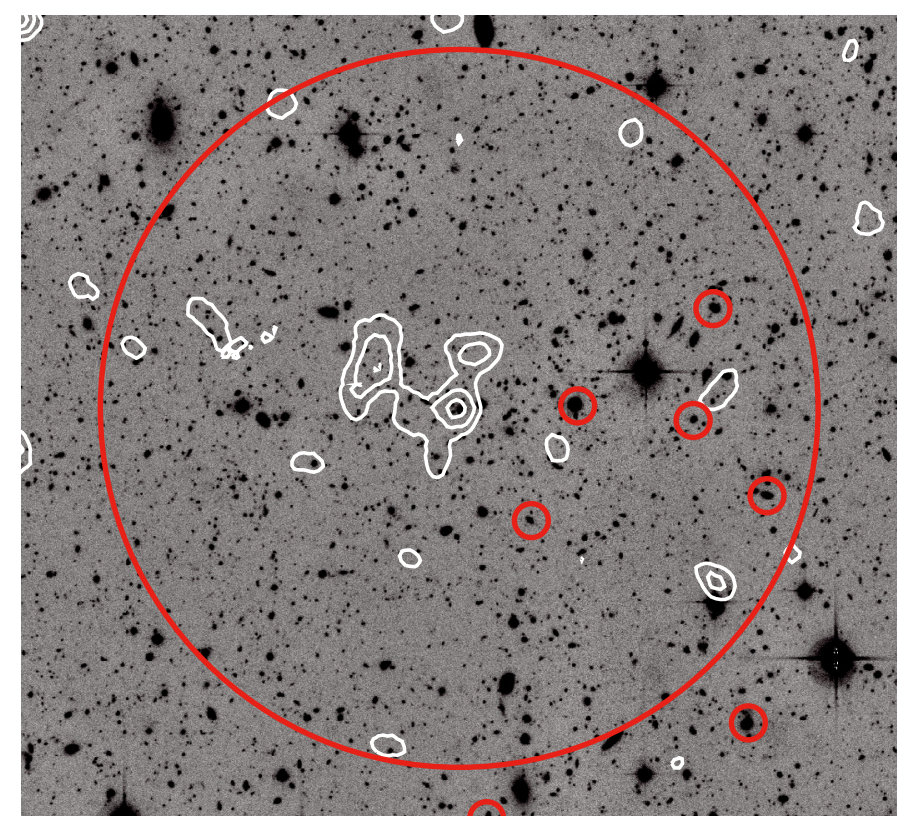

Fig. 5. CFHTLS $i^{\prime}$ band image of D3-6 overlaid with Chandra contours in white (see text). Small red circles show the galaxies with known spectroscopic redshifts inside the $z=0.607$ D3-6 structure. The large red circle represents a $1 \mathrm{Mpc}$ radius area.

ground-based seeing, it is still a difficult task to try to detect modest mass concentrations at $z \sim 0.74$, but we have no other possibility with the data available to tell if D3-43-S3 has a significant mass or not.

We cut a subimage centred on our structure with a field of view of $6.3^{\prime} \times 6.3^{\prime}$ (corresponding to $\left.2.74 \times 2.74 \mathrm{Mpc}^{2}\right)$. This is roughly the size of VLT/FORS2 images, which are well suited for cluster weak lensing studies at this redshift (e.g. Clowe et al. 2006). We measure object positions using SExtractor (Bertin \& Arnouts 1996) and shapes using the latest imcat software tools (Kaiser 2011). We then apply the standard KSB+ methodology for PSF correction (Kaiser et al. 1995; Luppino \& Kaiser 1997). The full detail of the method applied will be found in Martinet et al. (in prep.). The general idea is to measure the PSF distortion on the stars in the image to subtract it from the galaxy shape measurements. In practice, this information is retained in the fourth moment of object surface brightness distributions. Stars are discriminated from galaxies in a half-light radius versus magnitude plot. A visual inspection of both stars and galaxies in the field is mandatory to eliminate false detections, objects that are blended or near saturated stars and artefacts. We also apply a correction factor that represents the bias of our method and was calibrated on STEP2 simulations (Massey et al. 2007). We eliminate cluster and foreground galaxies to avoid diluting the signal, by considering photometric redshifts computed on the basis of $u^{*}, g^{\prime}, r^{\prime}$, $i^{\prime}, z^{\prime}, J, H, K$ s (CFHTLS and WIRDS release, see Bielby et al. 2012) magnitudes. Given the precision of these photometric redshifts, we remove all objects at redshifts lower than the structure redshift plus 0.1 , keeping only distant galaxies, the only ones potentially sensitive to the D3-43-S3 mass concentration. Finally, the shear catalogue is converted into a binned shear map, which is then inverted into a convergence map following Kaiser $\&$ Squires (1993). We then apply a Gaussian smoothing that allows us to estimate the noise level in the map (van Waerbeke 2000). The density of background galaxies is $\sim 18$ galaxies per square arcmin. The contours are given in numbers of sigma corresponding to the noise in the convergence map reconstruction.

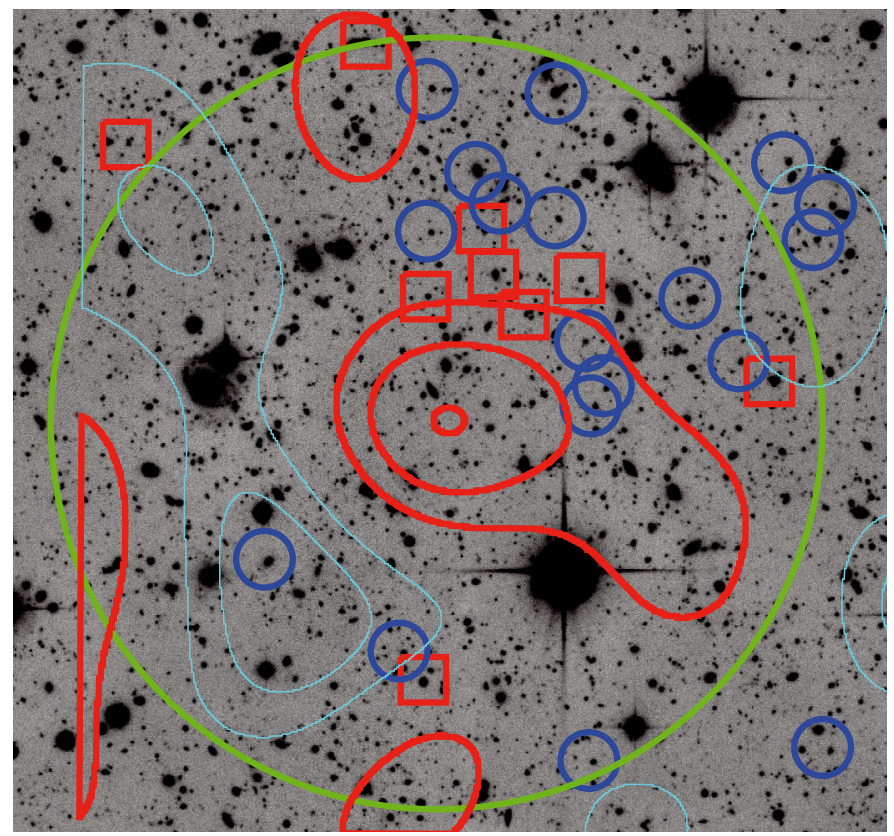

Fig. 6. CFHTLS $i^{\prime}$ band image of D3-43 overlaid with weak lensing contours (see text). Red squares and blue circles are the galaxies with known spectroscopic redshifts inside the $z=0.739$ D3-43-S3 substructures \#1 and \#2, respectively. The green circle has a radius of $1 \mathrm{Mpc}$. We show the positive sources as thick red contours and the negative sources as thin cyan contours. The contours are given in numbers of sigma corresponding to the noise in the convergence map reconstruction, starting with $1 \sigma$.

The result is that D3-43-S3 is barely detected at a $3 \sigma$ level (see Fig. 6). We note that given the quite low detection level, we did not try to calibrate the weak lensing countours in terms of mass.

We show in Fig. 6 the weak lensing contours and the galaxies with known spectroscopic redshifts in D3-43-S3 overlaid on the CFHTLS $i^{\prime}$ band image. Twentytwo of the galaxies with known spectroscopic redshifts are at less than $1 \mathrm{Mpc}$ from the cluster weak lensing centre. We will not discuss the weak lensing analysis of D3-6 since its detection level is smaller than $3 \sigma$.

\section{Properties of the galaxy populations in the two structures}

Publicly available photometric redshifts in the CFHTLS D3 field are among the best available from ground-based data (computed with LePhare, e.g. Coupon et al. 2009). Following Coupon et al. (2009), we chose to limit our photometric redshift samples to $i^{\prime} \leq 24\left(g^{\prime} \leq 25\right.$ with Fukugita et al. 1995).

We also verified that for the spectroscopic member galaxies of D3-43-S3, the statistical uncertainty of the photometric redshifts was of the order of 0.037 . To keep as many structure galaxies as possible in our samples, we then chose to select a slice of $\pm 3 \times 0.037$ around the two clusters. The price to pay is the likely inclusion of field galaxies satisfying this criterium. This contribution is quite easy to remove statistically however, using for example a comparison field (CF) taken in the CFHTLS D3 field (with the same angular size as the considered cluster), empty from any known candidate cluster and with galaxies selected in the same photometric redshift range.

The remaining uncertainty in the cluster galaxy counts comes from the catastrophic errors present in the photometric redshifts. To take this uncertainty into account, we considered 

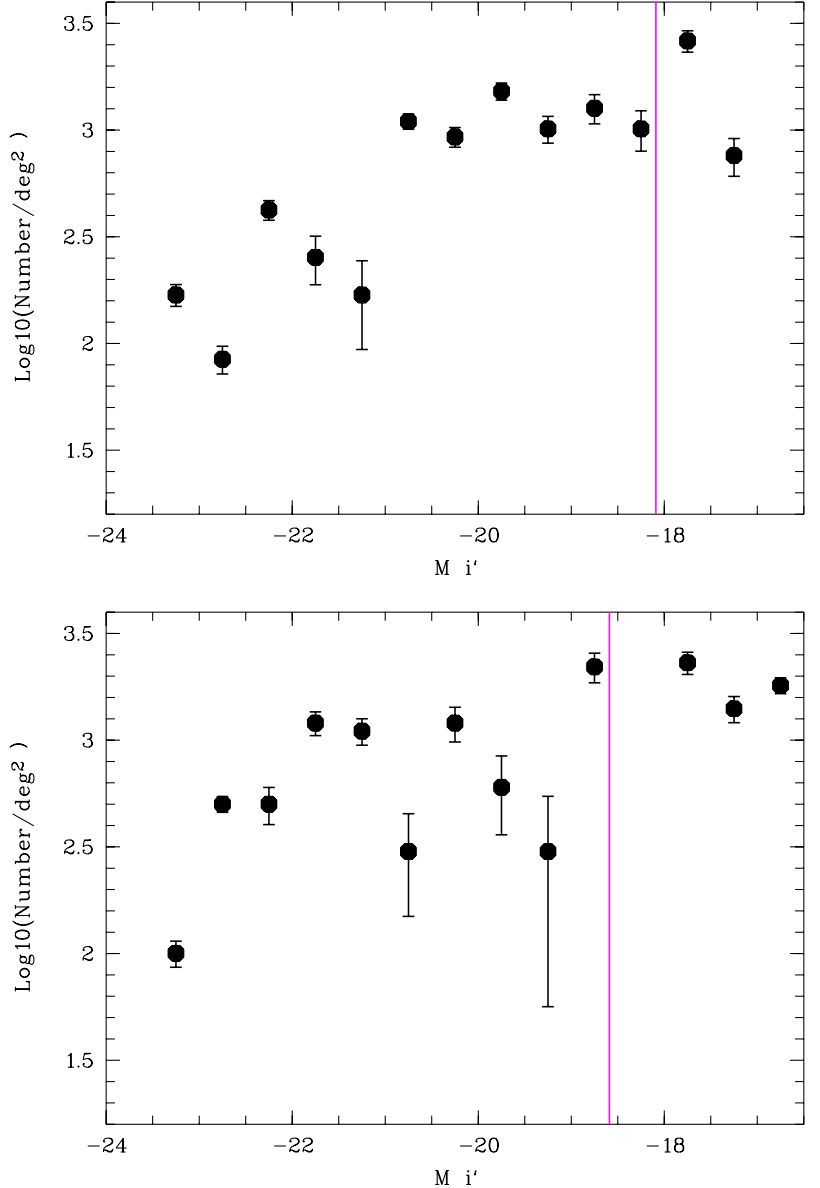

Fig. 7. Luminosity functions for the whole galaxy population of D3-6 (top) and D3-43-S3 (bottom) per bin of 0.5 mag normalised to $1 \mathrm{deg}^{2}$. The vertical lines show the limits in absolute magnitude computed from the $i^{\prime}=2490 \%$ completeness limit, applying the distance modulus and the mean K-correction in the D3-6 and D3-43-S3 fields.

the percentage of such catastrophic errors in the CFHTLS deep fields computed in Coupon et al. (2009): 4\%. This was added to the Poissonian error bars estimated for each magnitude bin in what follows.

We then re-ran the LePhare code with both the Cosmossurvey SEDs (e.g. Ilbert et al. 2010) and the Bruzual \& Charlot (2003) SEDs, fixing the redshifts of the selected samples to the spectroscopic redshifts of the two structures. We only considered galaxies for which all the bands were available. This gave us rest-frame absolute magnitudes, stellar masses, and star formation rates (SFRs).

\subsection{Galaxy luminosity functions}

Galaxy luminosity functions were computed by counting the galaxies along the D3-6 and D3-43-S3 lines of sight and statistically subtracting the empty field counts.

We then compared the rest-frame absolute $i^{\prime}$ magnitude band galaxy luminosity functions (in a $1.5 \mathrm{Mpc}$ radius) of the two clusters (Fig. 7). We note that K-corrections are already taken into account in the absolute magnitudes and this allows us to compare the luminosity functions of the two main structures. To compare the two luminosity functions, first we had to increase ( 0.07 towards the high values along the $y$-axis) the D3-6 values by $18 \%$ to take the fact that the two structures are at different
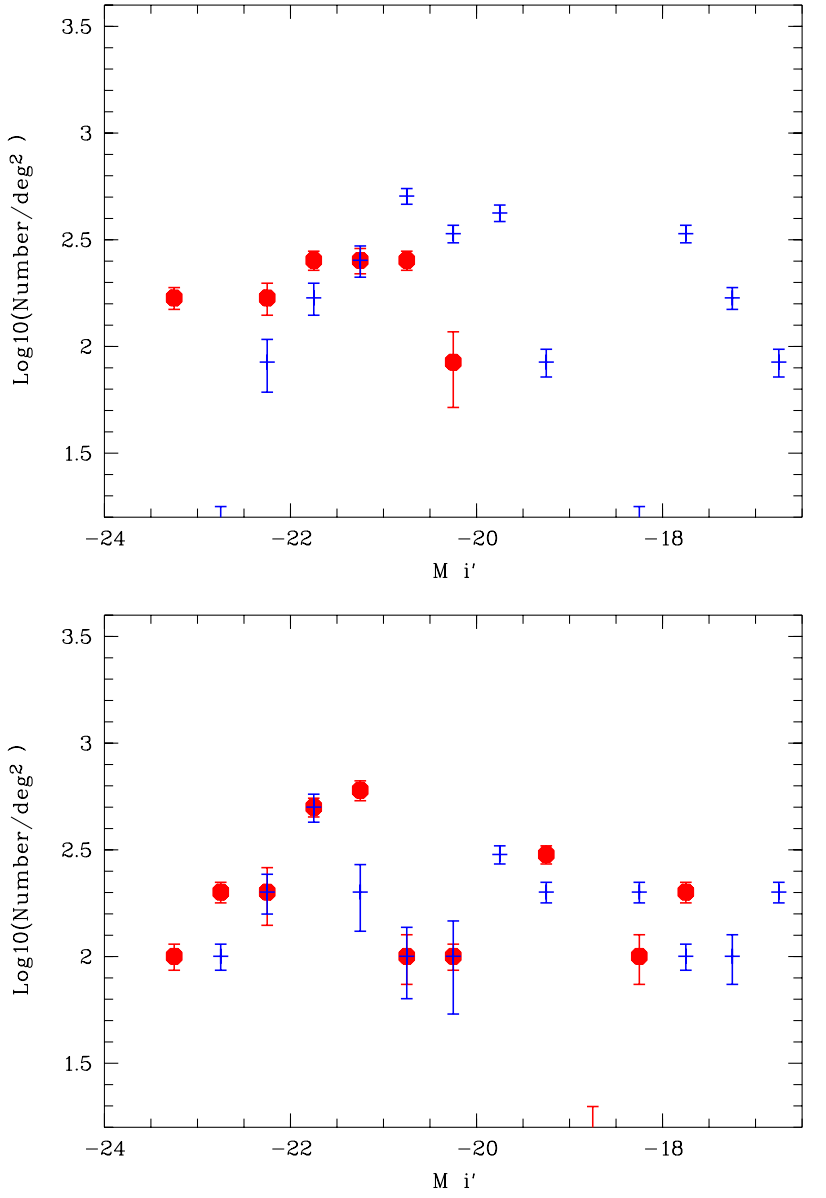

Fig. 8. Luminosity functions for the galaxies with morphological types classified as later (blue crosses) and earlier (red disks) than Sb. Upper panel: D3-6; lower panel: D3-43-S3.

redshifts into account. We limited the luminosity functions to absolute magnitudes brighter than -18.5 (see Fig. 7). We then performed 10000 realisations of the two luminosity functions within the error bars and performed a Kolmogorov-Smirnov test each time. The probability for the two luminosity functions to originate from the same parent distribution was then found to be higher than $99 \%$ for $47 \%$ of the realisations, and higher than $75 \%$ for $97 \%$ of the realisations. This clearly shows that the D36 and D3-43 luminosity functions are not significantly different.

The next step is to consider the luminosity functions per morphological type. This can be done by considering the modelled types available in the photometric redshift catalogues. These types go from 1 (elliptical galaxies) to 31 (starburst galaxies). We divided our galaxy sample into types earlier and later than Sb to generate Fig. 8 .

We performed the same Kolmogorov-Smirnov tests and found that for the D3-6 cluster, the probability for the early and late-type galaxy luminosity functions to originate from the same parent distribution was higher than $99 \%$ for $0 \%$ of the realisations, and higher than $75 \%$ for only $63 \%$ of the realisations. In this case, we can conclude that the early and late-type galaxy luminosity functions in D3-6 are probably different, and that this cluster clearly has more late-type galaxies than early-type galaxies in the faint magnitude regime (this is quite obvious in Fig. 8).

The same exercise yields different results for the D3-43-S3 cluster. The probability that the early and late-type galaxy luminosity functions originate from the same parent distribution 

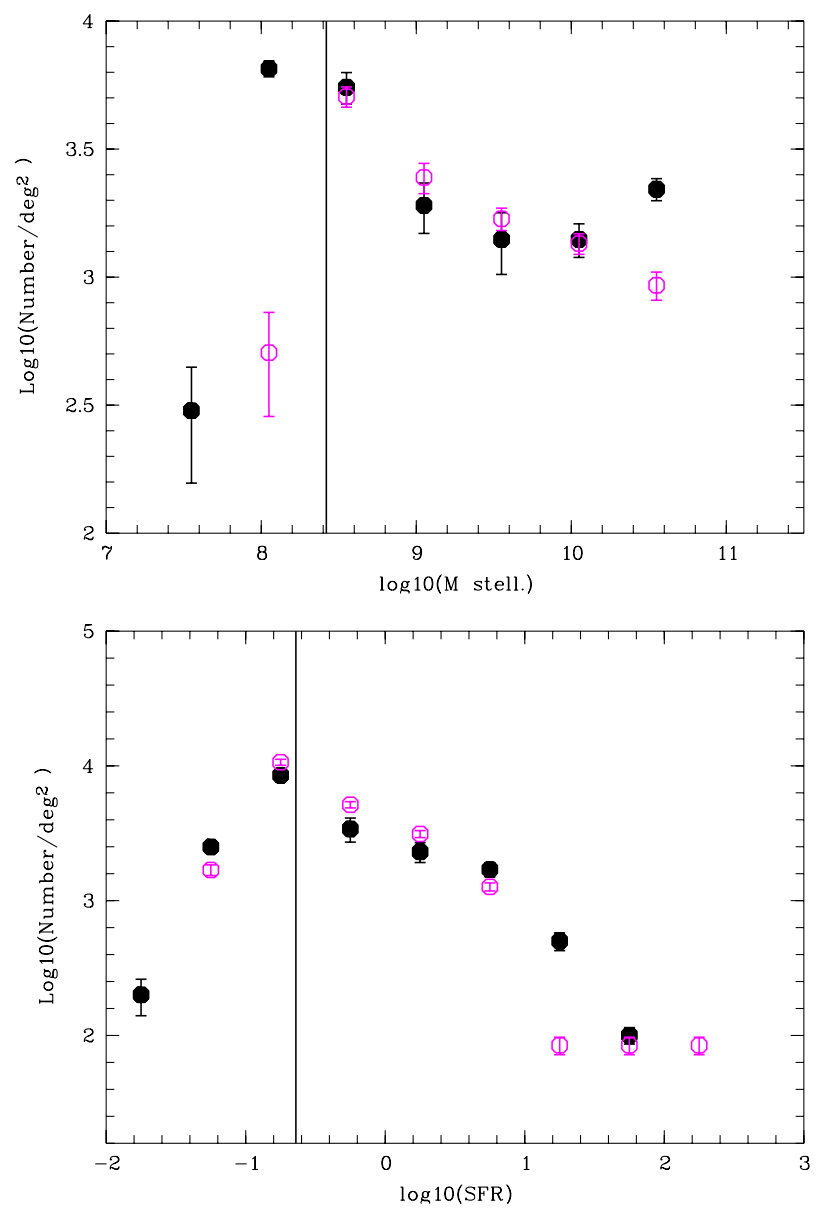

Fig. 9. Upper panel: stellar mass functions for the galaxy populations of D3-6 (pink circles) and D3-43-S3 (black disks). Lower panel: SFR functions for the galaxy populations of D3-6 (pink circles) and D3-43-S3 (black disks), in units of $M_{\odot} /$ yr. In both plots, the vertical lines show the values corresponding to the most stringent $i^{\prime}$ band absolute magnitude $90 \%$ completeness level.

is higher than $99 \%$ for $42 \%$ of the realisations, and higher than $75 \%$ for $100 \%$ of the realisations. In this case, we have similar luminosity functions for early and late-type galaxies (as seen in Fig. 8).

\subsection{Star formation history in D3-6 and D3-43-S3}

To investigate the stellar formation history in the galaxies of the considered clusters more precisely, we first computed galaxy stellar mass functions in D3-6 and D3-43-S3 (after statistical subtraction of the empty field values). These are displayed in Fig. 9. The two clusters exhibit very similar stellar mass functions. Even if D3-43-S3 is richer than D3-6 for the highest stellar mass bin, a Kolmogorov-Smirnov test shows that the two global distributions (in the domain where the data are $90 \%$ complete) are the same.

We also considered the SFRs of the galaxies in D3-6 and D3-43-S3 (see Fig. 9). A Kolmogorov-Smirnov test shows that the SFRs are very similar for both structures: probability greater than $99 \%$ for $49 \%$ of the realisations, and higher than $75 \%$ for $100 \%$ of the realisations. Considering each bin of Fig. 9 separately, the only significant differences occur towards high values of the SFR, with D3-43-S3 having more galaxies with SFR of $\sim 16 M_{\odot} / \mathrm{yr}$ (these galaxies could have their SFR boosted by the ongoing merger taking place in D3-43-S3), and D3-6 having more galaxies with intense SFR $\left(\sim 150 M_{\odot} / \mathrm{yr}\right)$.

We estimated the SFR and stellar mass completeness levels in the following way: we selected the galaxies with a magnitude differing by less than 0.1 from the $i^{\prime}$ magnitudes at the $90 \%$ completeness limit. We then computed the mean SFRs and stellar masses for these galaxies and considered that these values represented the SFR and stellar mass completeness levels.

\section{Conclusions}

With data obtained with GEMINI/GMOS and data taken from the literature and NED, we spectroscopically confirmed two structures initially detected with the AMACFI photometric redshift based cluster finder in the CFHTLS D3 field.

The first structure, D3-43-S3, can be decomposed into two substructures, each with a velocity dispersion of $\sim 350$ and $\sim 600 \mathrm{~km} \mathrm{~s}^{-1}$, which could be in the process of collapsing into a large structure more or less along the line of sight. Since no $\mathrm{X}$-ray emission is detected, the interaction is probably not strong yet, explaining the fact that we (barely) detect it in weak lensing (where we are probably seeing the addition of the two structure masses along the line of sight) but not in X-rays.

The D3-6 structure is found to be a single structure at an average redshift $z=0.607$, with a velocity dispersion of $423 \mathrm{~km} \mathrm{~s}^{-1}$. It appears to be a relatively low-mass cluster.

We also show that D3-6 and D3-43-S3 have similar global galaxy luminosity functions, stellar mass functions, and SFR distributions. The only differences are that D3-6 exhibits a lack of faint early-type galaxies, a deficit of extremely high stellar mass galaxies compared to D3-43-S3, and an excess of very high SFR galaxies.

Besides the fact that this work adds two compact galaxy structures to the strategical and still relatively poorly clusterpopulated $0.5<z<1$ redshift range, it also shows the power of photometric redshift based techniques to detect and study distant clusters (galaxy types, SFR, etc.), provided that a large spectral coverage in the optical and near infrared is available. Combined-approach cluster surveys (e.g. photometric redshifts to detect them and X-rays or weak lensing to characterise them in terms of mass) are also crucial. EUCLID is the perfect example of such a mission for clusters, since it will combine weak lensing and cluster detections based on photometric redshifts.

Acknowledgements. Based on observations obtained at the Gemini Observatory, which is operated by the Association of Universities for Research in Astronomy, Inc., under a cooperative agreement with the NSF on behalf of the Gemini partnership: the National Science Foundation (United States), the Science and Technology Facilities Council (United Kingdom), the National Research Council (Canada), CONICYT (Chile), the Australian Research Council (Australia), Ministério da Ciência, Tecnologia e Inovação (Brazil) and Ministerio de Ciencia, Tecnología e Inovación Productiva (Argentina). This research has made use of the VizieR catalogue access tool at CDS, Strasbourg, France. This research has also made use of the NASA/IPAC Extragalactic Database (NED), which is operated by the Jet Propulsion Laboratory, California Institute of Technology, under contract with the National Aeronautics and Space Administration. Finally, this paper is based on observations obtained with MegaPrime/MegaCam, a joint project of CFHT and CEA/IRFU, at the Canada-France-Hawaii Telescope (CFHT), which is operated by the National Research Council (NRC) of Canada, the Institut National des Sciences de l'Univers of the Centre National de la Recherche Scientifique (CNRS) of France, and the University of Hawaii. This work is based in part on data products produced at Terapix available at the Canadian Astronomy Data Centre as part of the Canada-France-Hawaii Telescope Legacy Survey, a collaborative project of NRC and CNRS. We would like to dedicate this paper to Alain Mazure, deceased in 2013, and who was part of this work in its early stages. We gratefully acknowledge financial support from the Centre National d'Etudes Spatiales during many years. This work was also supported by the Brazilian agencies FAPESP and CNPq and benefited 
from the CAPES-COFECUB agreement number 711/11. We thank the referee for interesting comments.

\section{References}

Adami, C., Biviano, A., Durret, F., \& Mazure, A. 2005, A\&A, 443, 17 Adami, C., Le Brun, V., Biviano, A. et al. 2009, A\&A, 507, 1225

Adami, C., Durret, F., Benoist, C., et al. 2010, A\&A, 509, A81 Adami, C., Mazure, A., Pierre, M., et al. 2011, A\&A, 526, A18 Beers, T. C., Gebhardt, K., Forman, W., Huchra, J. P., \& Jones, C. 1991, AJ, 102, 1581

Bertin, E., \& Arnouts, S. 1996, A\&AS, 117, 393

Bielby, R., Hudelot, P., McCracken, H. J., et al. 2012, A\&A, 545, A23

Boué, G., Durret, F., Adami, C., et al. 2008, A\&A, 489, 11

Boselli, A., Voyer, E., Boissier, S., et al. 2014, A\&A, 570, A69

Bruzual, G., \& Charlot, S. 2003, MNRAS, 344, 1000

Clowe, D., Schneider, P., Aragón-Salamanca, A., et al. 2006, A\&A, 451, 395

Coupon, J., Ilbert, O., Kilbinger, M., et al. 2009, A\&A, 500, 981

Dressler, A., \& Schechtman, S. A. 1988, AJ, 95, 985

Dunkley, J., Komatsu, E., Nolta, M. R., et al. 2009, ApJS, 180, 306

Durret, F., Adami, C., Gerbal, D., \& Pislar, V. 2000, A\&A, 356, 815

Durret, F., Slezak, E., \& Adami, C. 2009, A\&A, 506, 637

Durret, F., Laganá, T. F., Adami, C., \& Bertin, E. 2010, A\&A, 517, A94

Erfanianfar, G., Finoguenov, A., Tanaka, M., et al. 2013, ApJ, 765, 117

Fukugita, M., Shimasaku, K., \& Ichikawa, T. 1995, PASP, 107, 945

Garilli, B., Fumana, M., Franzetti, P., et al. 2010, PASP, 122, 827

Guennou, L. 2012, Ph.D., Université de Marseille-Provence

Guennou, L., Adami, C., Durret, F., et al. 2014, A\&A, 561, A112

Guzzo, L., Scodeggio, M., Garilli, B., et al. 2014, A\&A, 566, A108
Holden, B. P., Illingworth, G. D., Franx, M., et al. 2007, ApJ, 670, 190 Howell, D. A., Sullivan, M., Perrett, K., et al. 2005, ApJ, 634, 1190 Hsieh, B. C., Yee, H. K. C., Lin, H., \& Gladders, M. D. 2005, ApJS, 158, 161

Ilbert, O., Arnouts, S., McCracken, H. J., et al. 2006, A\&A, 457, 841

Ilbert, O., Salvato, M., Le Floc'h, E., et al. 2010, ApJ, 709, 644

Kaiser, N., \& Squires, G. 1993, ApJ, 404, 441

Kaiser, N., Squires, G., \& Broadhurst, T. 1995, ApJ, 449, 460

Kaiser, N. 2011 [record ascl: 1108.001]

Katgert, P., Mazure, A., Perea, J., et al. 1996, A\&A, 310, 8

Lopes, P. A. A., de Carvalho, R. R., Kohl-Moreira, J. L., \& Jones, C. 2009, MNRAS, 399, 2201

Luppino, G. A., \& Kaise., N. 1997, ApJ, 475, 20

Massey, R., Heymans, C., Bergé, J., et al. 2007, MNRAS, 376, 13

Mateus, A., Sodré, L., Cid Fernandes, R., \& Stasińska, G. 2007, MNRAS, 374, 1457

Mazure, A., Adami, C., Pierre, M., et al. 2007, A\&A, 467, 49

Newman, J. A., Cooper, M. C., Davis, M., et al. 2013, ApJS, 208, 5

Olsen, L. F., Benoist, C., Cappi, A., et al. 2007, A\&A, 461, 81

Poggianti, B. M., De Lucia, G., Varela, J., et al. 2010, MNRAS, 405, 995

Ross, A. J., Percival, W. J., \& Brunner, R. J. 2010, MNRAS, 407, 420

Sánchez-Blázquez, P., Jablonka, P., Noll, S., et al. 2009, A\&A, 499, 47

Serna, A., \& Gerbal, D. 1996, A\&A, 309, 65

Simard, L., Clowe, D., Desai, V., et al. 2009, A\&A, 508, 1141

Thanjavur, K., Willis, J., \& Crampton, D. 2009, ApJ, 706, 571

Ulmer, M. P., Adami, C., Lima Neto, G. B., et al. 2009, A\&A, 503, 399

van der Wel, A., Holden, B. P., Franx, M., et al. 2007, ApJ, 670, 206

van Waerbeke, L. 2000, MNRAS, 313, 524

Walker, E. S., Hook, I. M., Sullivan, M., et al. 2011, MNRAS, 410, 1262

White, S. D. M., \& Frenk, C. 1991, ApJ, 379, 52 


\section{Appendix A: DEIMOS/GMOS redshift comparison}

We show in Fig. A.1 the difference between the GEMINI/GMOS redshifts and the KECK/DEIMOS DEEP2 redshifts as a function of redshift.

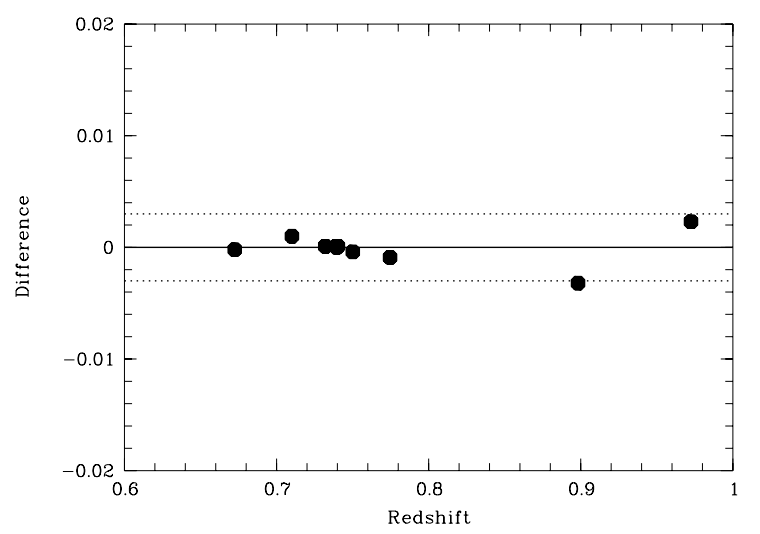

Fig. A.1. Comparison of Deep2 Keck/DEIMOS and GMOS/Gemini redshifts. The solid horizontal line symbolises the perfect agreement. The two dashed lines represent the $3 \sigma$ maximum uncertainty between the two redshift measurements taking the resolution of the GMOS grism and the smoothing of the spectra into account.

\section{Appendix B: Examples of spectra}

Below are four examples of spectra corresponding to flags 4, 3, and 2 in Figs. B.1 and B.2.
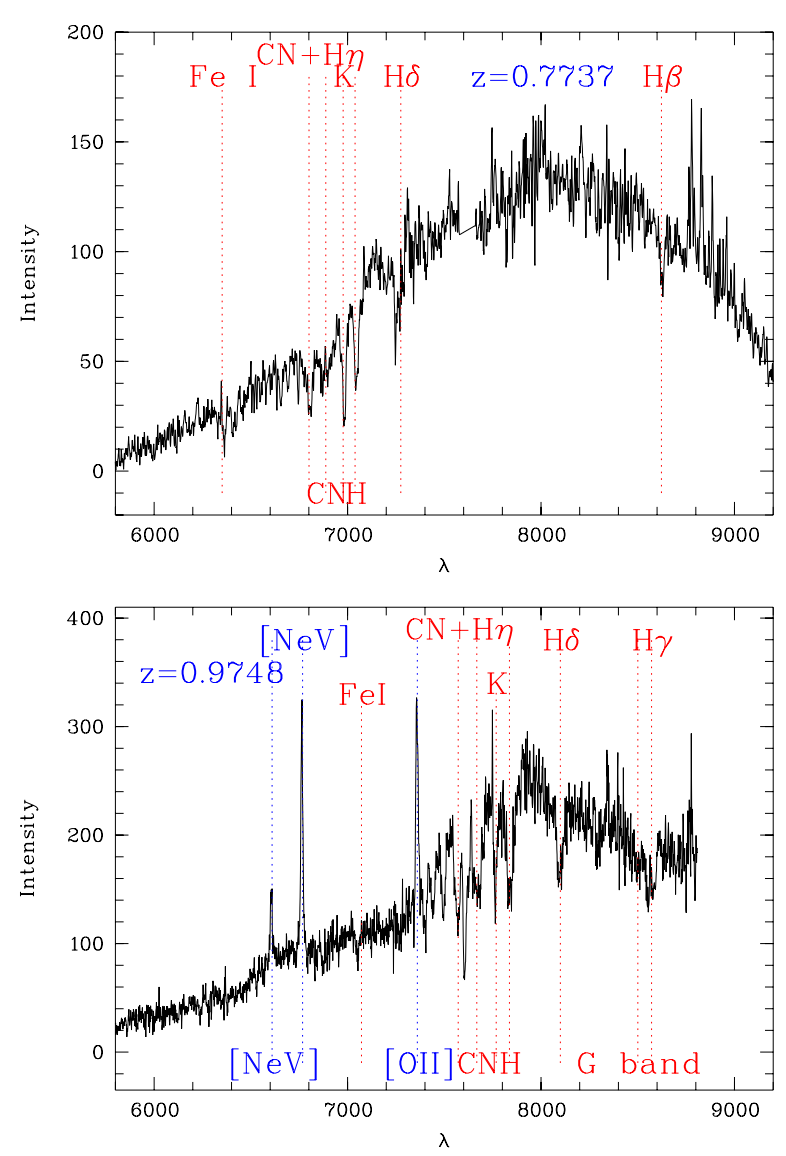

Fig. B.1. Two examples of Gemini/GMOS spectra with flags 4. Red labelled lines show absorption lines and blue labelled lines show emission lines.

A69, page 10 of 10
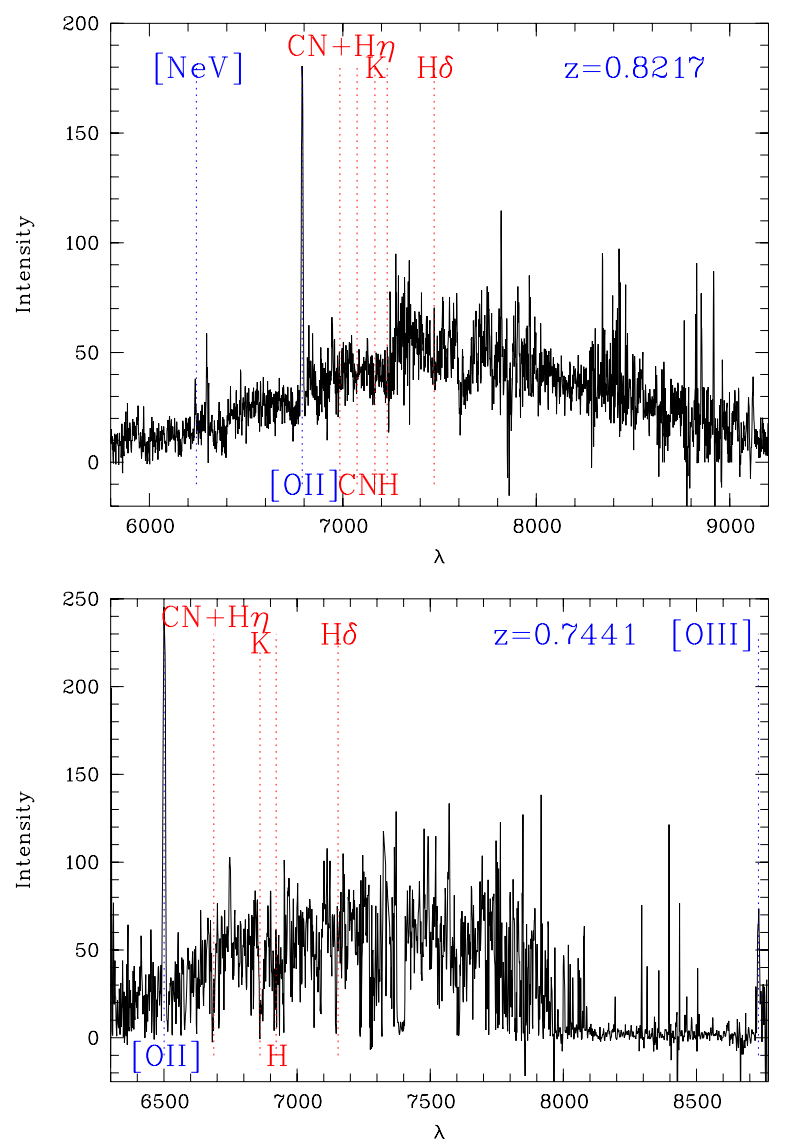

Fig. B.2. Two examples of Gemini/GMOS spectra with flags 3 and 2. Red labelled lines show absorption lines and blue labelled lines show emission lines. From top to bottom: one spectrum with flag 3 and one spectrum with flag 2 . 